\title{
Shielding and activation calculations around the reactor core for the MYRRHA ADS design
}

\author{
Anna Ferrari ${ }^{\mathrm{a}, 1}$, Stefan Mueller ${ }^{1}$, J. Konheiser ${ }^{1}$, D. Castelliti ${ }^{2}$, M. Sarotto $^{3}$ and A. Stankovskiy ${ }^{2}$ \\ ${ }^{1}$ Helmholtz-Zentrum Dresden-Rossendorf, PF 910119, 01314 Dresden, Germany \\ ${ }^{2}$ SCK.CEN, Boretang 200, $2400 \mathrm{Mol}$, Belgium \\ ${ }^{3}$ ENEA, Via Martiri di Monte Sole 4, 40129 Bologna, Italy
}

\begin{abstract}
In the frame of the FP7 European project MAXSIMA, an extensive simulation study has been done to assess the main shielding problems in view of the construction of the MYRRHA accelerator-driven system at SCK CEN in Mol (Belgium). An innovative method based on the combined use of the two state-of-the-art Monte Carlo codes MCNPX and FLUKA has been used, with the goal to characterize complex, realistic neutron fields around the core barrel, to be used as source terms in detailed analyses of the radiation fields due to the system in operation, and of the coupled residual radiation. The main results of the shielding analysis are presented, as well as the construction of an activation database of all the key structural materials. The results evidenced a powerful way to analyse the shielding and activation problems, with direct and clear implications on the design solutions.
\end{abstract}

\section{Introduction}

Accelerator-driven systems (ADS) are one of the options studied for the transmutation of nuclear waste in the international community. The MYRRHA [1] facility at SCK.CEN in Mol (Belgium) aims to demonstrate efficient transmutation of high level waste and associated ADS technology. The heart of the system is a leadbismuth eutectic (LBE) cooled reactor, working both in critical and in sub-critical operation modes. The neutrons needed to sustain fission in the sub-critical mode are produced via spallation processes by a $600 \mathrm{MeV}, \leq 4 \mathrm{~mA}$ proton beam, which is provided by a linear accelerator and hits, along a vertical beamline, a LBE spallation target located inside the reactor core. The combination with a nuclear reactor core makes the shielding problem an issue, being the protection from the prompt radiation and the spent beam handling the main points. Due to the backscattered neutron radiation on the spallation target, the operation in sub-critical mode drives the shielding in all the vertical structures, and requires more innovative solutions. This work is therefore focused on this operation mode.

\section{Method}

A systematic analysis has been done using two state-ofthe-art Monte Carlo codes: MCNPX [2], version 2.6.0, and FLUKA [3, 4], version 2011.2. As first step, an accurate MCNPX model of the reactor sub-critical core has been developed, in agreement with the most recent MYRRHA design. To simulate high energy processes, the Cascade-Exciton model, CEM03 [5], has been used. Neutron radiation fields have been then fully characterized on suitable surfaces around the core barrel and at the top of the fuel elements and used to build complex source terms, to be used in a second row of FLUKA simulations. A precious advantage of FLUKA is the possibility to evaluate, in the same simulation, the radiation fields due to the system in operation and the ones due to the coupled residual radiation. This is performed in the code by a half-analytic/half- Monte Carlo method, which is shortly summarized in the following. For a given irradiated material, the time evolution of its isotopic densities, $N_{i}$, is given by the set of the Bateman equations:

$$
\frac{d N_{i}}{d t}=-\sum_{j \neq i}\left[\lambda^{d}+\bar{\sigma}_{j i} \bar{\varphi}\right]_{N_{i}}+\sum_{j \neq i}\left[\lambda_{i j}^{d}+\bar{\sigma}_{i j} \bar{\varphi}\right] N_{j}
$$

where $\lambda_{j i}^{d}$ is the decay probability of the radionuclide $i$ in the radionuclide $j$ and $\sigma_{j i}$ is the particle induced cross section for transmutation from the isotope $i$ to the isotope $j$, and where $\bar{\varphi}$ and $\bar{\sigma}_{j i}$ are the average spectrum and the spectrum averaged, particle induced crosssection, respectively:

$$
\bar{\varphi}=\int \varphi(E) d E, \quad \bar{\sigma}_{j i}=\frac{1}{\bar{\varphi}} \int \varphi(E) \sigma_{j i}(E) d E
$$

In the Eq. (1) second terms in brackets represent the radionuclide production due to the irradiation, and in FLUKA are evaluated runtime via Monte Carlo. First terms represent on the other hand the decay contribution

\footnotetext{
a Corresponding author: a.ferrari@hzdr.de
} 
to the radionuclide equilibrium, and are analytically computed. Once the Bateman equations are solved, in the same run FLUKA performs the generation and transport of the emitted residual radiation with a full Monte Carlo method. This means that in the same run we can obtain the production of the residuals, their time evolution and the residual dose due to their decay.

To characterize the structures around the core, the main primary system components have been represented with a simplified geometry and a more homogeneous material composition. Minor internal elements have been neglected and all components have been described through a radial and axial homogenization. The resulting structure maintains a high level of complexity and includes the reactor vessel, the diaphragm and the cover model, the LBE and the gas plenum above, a homogenization of the structures above the core, the fuel handling machinery, the primary heat exchangers and the primary pumps. Beyond the reactor cover, a simplified geometrical model extends to include all the vertical part of the proton beamline, until the final roof.

\section{Results}

\subsection{Shielding}

Neutron and photon radiation fields and ambient dose equivalent distributions have been computed in all the structures around the core. Fig. 1, Fig. 2 and Fig. 3 show an example of neutron and photon fluence rate distributions, as well as of the $H^{*}(10)$ rate. The photon contribution of Fig. 2 is not due to photons coming from the reactor, but to the interactions of neutrons in the invessel structures, and - if we neglet the secondary radiation possibly coming from the beam losses inside the proton beam-pipe - is the only photon contribution that affects the dose rate far from the core. The present study had an impact on the design of the cover, which is in borated water contained in a steel structure. It can be observed that neutrons are very well absorbed in the borated water, while on the other hand the neutron capture processes on hydrogen and on boron generate

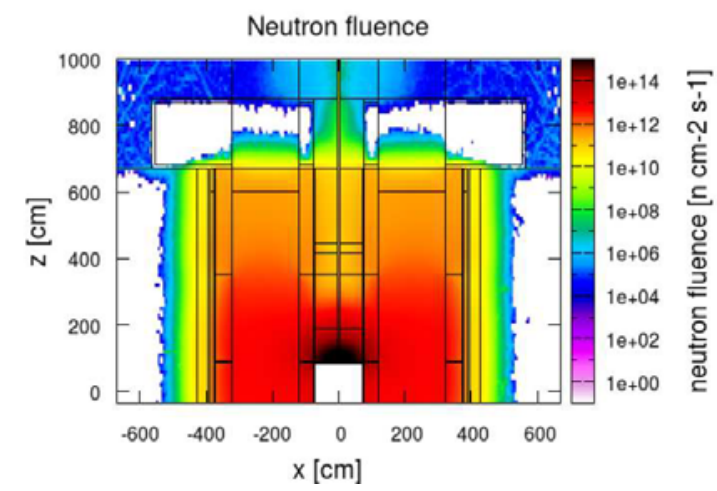

Figure 1. Neutron fluence rate [neutrons $\mathrm{cm}^{-2} \mathrm{~s}^{-1}$ ] in the invessel structures, until the cover plate.

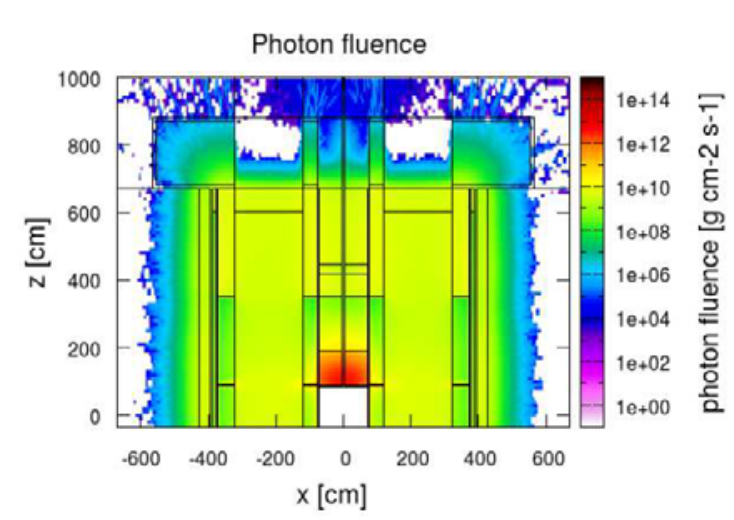

Figure 2. Photon fluence rate $\left[\right.$ photons $\left.\mathrm{cm}^{-2} \mathrm{~s}^{-1}\right]$ in the in-vessel structures.

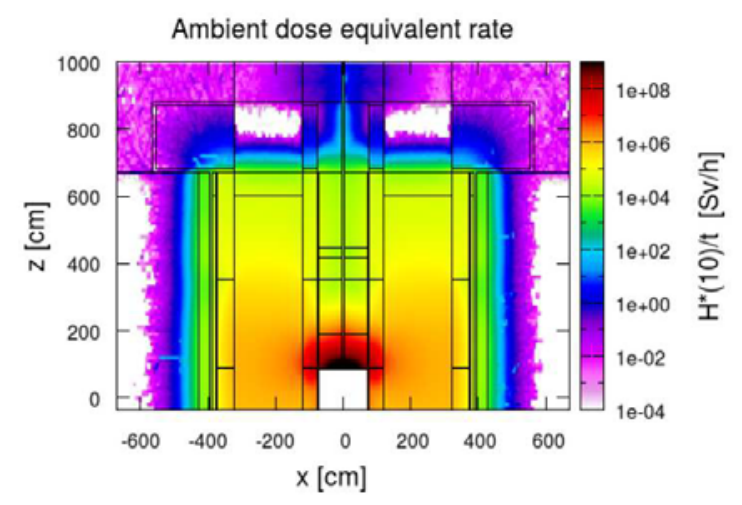

Figure 3. $\mathrm{H}^{*}(10)$ rate $[\mathrm{Sv} / \mathrm{h}]$ in the in-vessel structures, including all contributions to the radiation.

photons in the $\mathrm{MeV}$ range, which are not perfectly contained by the steel top cover plate (see Fig. 2). To fully contain this radiation and consolidate an already effective design, a thicker containment structure is planned.

\subsection{Activation}

An extensive analysis of the residual radiation has been performed. The operation of the system has been conservatively considered in a scheme of 3-months cycles in the sub-critical mode at the maximum beam intensity $\left(\mathrm{I}_{\text {prot }}=4 \mathrm{~mA}\right)$, followed by 30 days stop. This irradiation profile has been implemented in the FLUKA radiation model and extended over 40 years, which is the planned MYRRHA life. The residual ambient dose equivalent rates in the system and 62 key regions selected for activation studies have been analysed for several points in time during the cooling, going from the end of the first cycle (EOC) until the end of the reactor life (EOL), which is assumed to be 40 years. An additional study of the residual radiation until a cooling of $10^{4}$ years will be also presented.

In Fig. 4 the results for the residual dose rates in the structures around the core are reported for a 
short/medium irradiation period (90 days sub-cycle), with a cooling time of 1 week. It can be observed the strong activation of the structures above the core, which contain high fractions of steel, especially in the hottest region above the core. The data also show an important residual dose rate in the nitrogen gas plenum, due to the activation of nitrogen from the reaction $14 \mathrm{~N}(\mathrm{n}, \mathrm{p}) 14 \mathrm{C}$, which exhibits a very high neutron capture cross-section (1.83 b) in the thermal region. It has to be noticed that the total ${ }^{14} \mathrm{C}$ production would be in any case largely below the common regulatory limits, as reported by IAEA [6].

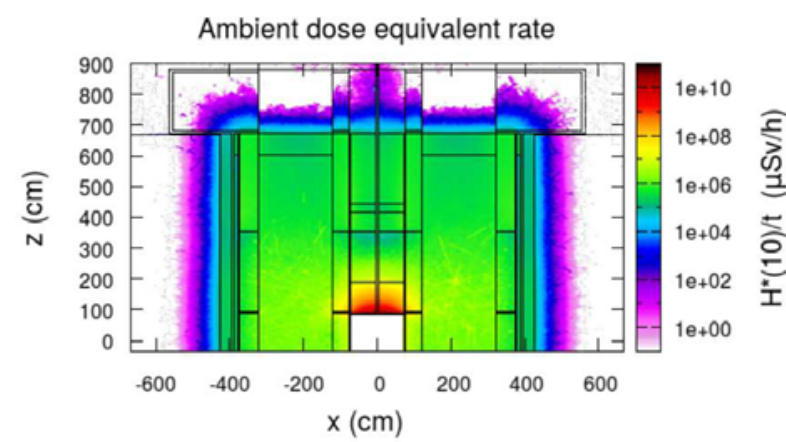

Figure 4. $H^{*}(10)$ residual dose rate $[\mu \mathrm{Sv} / \mathrm{h}]$ after 90 days of irradiation and 1 week cooling.

As example of the activation study after the end of the MYRRHA life, the residual activity in the diaphragm, the vessel and the liner are shown in Fig.5, for very long times (until $10^{4} \mathrm{y}$ ) after the end of the reactor operation.

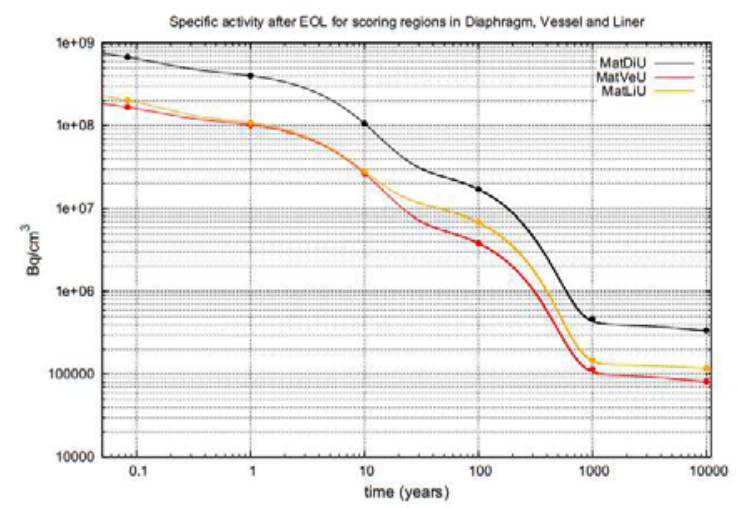

Figure 5. Residual activity $\left[\mathrm{Bq} / \mathrm{cm}^{3}\right]$ in the Diaphragm, the Vessel and the Liner in the most irradiated region.

\section{References}

1. D. De Bruyn, H. Aït Abderrahim, P. Baeten and R. Fernandez, MYRRHA, the Multi-purpose Hybrid Research Reactor for High-tech Applications, Proc. Int. Congress on Advances in NPPs (ICAPP '11),
Nice, France, May 2-6, 2011, France Omnipress, 472-478 (2011).

2. MCNPX 2.6.0 Manual, LANL Report LA-CP-071473 (2008).

3. A. Ferrari, P. Sala, A. Fassò, J.Ranft, "FLUKA: A multi-particle transport code", CERN-2005-10 (2005), INFN/TC_05/11, SLAC-R-773.

4. G. Battistoni, S. Muraro, P.R. Sala, F. Cerutti, A. Ferrari, S. Rösler, A. Fassò, J. Ranft, "The FLUKA code: description and benchmarking", Proc. Hadronic Shower Simulation Workshop 2006, Fermilab 6-8 September 2006, AIP Conference Proceedings, 896, 31-49 (2007).

5. S.G. Mashnik, K.K. Gudima, R.E. Prael, A.J. Sierk, M.I. Baznat and N.V. Mokhov, "CEM03.03 and LAQGSM03.03 event generators for the MCNP6, MCNPX, and MARS15 transport codes", LANL Report LA-UR-08-2931 (2008).

6. IAEA Technical Reports Series No. 421, Management of waste containing tritium and carbon14,Vienna (2004). 